\title{
Localization in Rotational Excitation of Diatomic Molecules Induced by a Train of Optical Pulses
}

\author{
Leo MATSUOKA ${ }^{1, *}$ and Etsuo SEGAWA ${ }^{2}$ \\ ${ }^{1}$ Graduate School of Engineering, Hiroshima University, Higashi-Hiroshima 739-8527, Japan \\ ${ }^{2}$ Graduate School of Information Sciences, Tohoku University, Sendai 980-8579, Japan
}

\begin{abstract}
We theoretically investigate the localization of population distribution in rotational excitation of diatomic molecules induced by a train of optical pulses in the terahertz region. In a simulation with parameters of real molecules, the localization is observed as a combined effect of several causes. For mathematical analysis, we classify the localization into four types based on the viewpoints of physical processes. We provide some extreme numerical examples of the four types of localizations.
\end{abstract}

KEYWORDS: quantum walks, quantum kicked rotor, isotope separation, nuclear wastes

\section{Introduction}

Massive and efficient rotational excitation of diatomic molecules can be achieved by irradiating an optical pulse train whose intervals are precisely tuned to the period of molecular rotation [1]. This effect is called quantum resonance [2]. We can select one isotope from an isotopic mixture by tuning the pulse interval, as the period of molecular rotation depends on each isotope [3-5]. In the condition of ideal quantum resonance, the molecules at any initial rotational state will be rotationally excited and de-excited, and eventually they all will be transferred into highly rotationally excited states [6]. This process has a possibility to be applied for highly efficient isotope separation in radioactive nuclear wastes $[7,8]$. However, as long as we use conventional methods, the ideal quantum resonance cannot be realized in real molecules. The localization of population distribution will inevitably be induced by the centrifugal distortion of molecules and imperfections of the optical pulses. To implement efficient isotope separation by rotational excitation, we have to develop a fundamental theory to predict the range of localization. This range can be easily evaluated by numerical simulations of time-dependent Schrödinger equation systems. However, we cannot easily reach optimized parameters for industrial applications using huge amounts of repetition of numerical calculations. This is because the localization would be observed as a combined effect of several causes in calculations using real parameters from experiments. To obtain a systematic parameter design method for industrial applications, we have to develop a mathematical foundation and analyze each localization process independently. This foundation will provide reasonable experimental parameters that could not be obtained by the repetition of numerical calculations.

The molecular rotational excitation by optical pulses at the most ideal condition can be described as the continuoustime quantum walk (CTQW) [9]. We regard the molecular excitation as an extended class of CTQWs, and try to apply some mathematical methods developed for quantum walks to the present system. In a previous report, we proposed a mathematical model of molecular rotational excitation and derived a unified parameter to predict localization behavior and its dependence on the pulse amplitude under some simplifying assumptions [10]. In this paper, we introduce further general model equations and classify the types of localization from the viewpoint of physics. This classification was performed by numerical observation and physics considerations; however, the provision of some analytically-solvable simple cases would accelerate the mathematical research on quantum walks. Finally, we discuss a prospect towards the unification of the presented localizations.

\section{Mathematical Model}

The mathematical modeling of molecular rotational excitation has been described in our previous report [10]. In the previous work, we assumed a constant transition probability between neighboring states for simplicity. Here, we eliminate this assumption to work with general cases. The time-dependent Schrödinger equation system for molecular rotational excitation is given by:

Received March 29, 2016; Accepted October 2, 2016

2010 Mathematics Subject Classification: Primary 81V55, Secondary 82C20.

This work is supported by JSPS KAKENHI Grant No. JP26420875.

*Corresponding author. E-mail: leo-matsuoka@hiroshima-u.ac.jp 


$$
i \frac{d}{d t} C_{J M}(t)=-\varepsilon(t)\left[\mu_{J-1, M} \exp \left\{\left(E_{J}-E_{J-1}\right) i t\right\} C_{J-1, M}(t)+\mu_{J M} \exp \left\{\left(E_{J}-E_{J+1}\right) i t\right\} C_{J+1, M}(t)\right]
$$

where $C_{J M}(t)$ is the complex amplitude of the rotational states $(J, M)$ at time $t, \varepsilon(t)$ is the electric field, $\mu_{J M}$ is the transition dipole moment from $(J, M)$ to $(J+1, M)$, and $E_{J}$ is the rotational energy of state $J$. $C_{J M}(t)$ and $\mu_{J M}$ are only defined for $J \geq 0$. For $J<0, \mu_{J M}$ is set to be zero. The probability distribution of state $J$ is given by $\left|C_{J M}(t)\right|^{2}$.

The rotational energy of molecules at each state $E_{J}$ is given by

$$
E_{J}=2 \pi B_{\mathrm{M}} J(J+1)-2 \pi D_{\mathrm{v}} J^{2}(J+1)^{2},
$$

where $B_{\mathrm{M}}$ and $D_{\mathrm{v}}$ are molecular spectroscopic constants for a rotational series. Here, we only deal with a single rotational series with fixed $M$ value by ignoring vibrational excitation and assuming a linear polarization of optical pulses.

A train of optical pulses is expressed by a summation of cosine waves as

$$
\varepsilon(t)=B_{\mathrm{f}} \varepsilon_{\mathrm{DC}}+2 B_{\mathrm{f}} \sum_{j=0}^{N-1} \varepsilon_{j} \cos \left\{4 \pi B_{\mathrm{f}}(j+1) t\right\},
$$

where $\varepsilon_{\mathrm{DC}}$ is the scaled amplitude of DC component, $\varepsilon_{j}$ is the scaled spectral amplitude inducing transition from $J=j$ to $J=j+1, B_{\mathrm{f}}$ is the frequency constant that corresponds to $B_{\mathrm{M}}$ of the molecules, and $N$ is the maximum $J$ state to be connected by optical transitions. The molecular classical rotational period and pulse repetition interval are given by $T_{\text {rot }}=\frac{1}{2 B_{\mathrm{M}}}$ and $T_{\text {rep }}=\frac{1}{2 B_{\mathrm{f}}}$, respectively.

By substituting Eq. (2.3) into Eq. (2.1), and performing the rotating-wave approximation, we can obtain the model equation system as

$$
\begin{aligned}
-i \frac{d}{d t} C_{J M}(t)= & B_{\mathrm{f}} \varepsilon_{J-1} \mu_{J-1, M} \exp \left\{4 \pi i t \Delta B J-8 \pi i t D_{\mathrm{v}} J^{3}\right\} C_{J-1, M}(t) \\
& +B_{\mathrm{f}} \varepsilon_{J} \mu_{J, M} \exp \left\{-4 \pi i t \Delta B(J+1)+8 \pi i t D_{\mathrm{v}}(J+1)^{3}\right\} C_{J+1, M}(t),
\end{aligned}
$$

where $\Delta B=B_{\mathrm{M}}-B_{\mathrm{f}}$. In the rotating-wave approximation, we consider only the most slowly changing terms in the equations. The correctness of the rotating-wave approximation was validated in $\epsilon_{J} \mu_{J M} \lesssim 5$ by numerical calculations in the previous report [10]. By dividing all the frequency parameters by $B_{\mathrm{f}}$, we obtain the model equation system as:

$$
\begin{aligned}
-i \frac{d}{d t} C_{J M}(t)= & \sqrt{\omega_{J}} \exp \left\{4 \pi i t \frac{\Delta B}{B_{\mathrm{f}}} J-8 \pi i t \frac{D_{\mathrm{v}}}{B_{\mathrm{f}}} J^{3}\right\} C_{J-1, M}(t) \\
& +\sqrt{\omega_{J+1}} \exp \left\{-4 \pi i t \frac{\Delta B}{B_{\mathrm{f}}}(J+1)+8 \pi i t \frac{D_{\mathrm{v}}}{B_{\mathrm{f}}}(J+1)^{3}\right\} C_{J+1, M}(t),
\end{aligned}
$$

where $\sqrt{\omega_{J+1}}=\varepsilon_{J} \mu_{J M}$.

\section{Classification of Localizations}

In the model system, the time evolution of the rotational population distribution is localized due to several reasons. We classify the localization into four types, and introduce them with extreme examples of numerical simulations. The classification was performed by considering the physical sources of localization.

\subsection{Localization 1: Localization by Interval Mismatching}

Even if the molecule can be regarded as a rigid rotor, the strong localization will be observed in the condition of $\Delta B \neq 0$. This localization comes from the interval mismatch between molecular rotation and pulse repetition, and is the main principle of isotope selection. The range of localization depends on $\Delta B$ and on the pulse amplitude, and it's prediction is non-trivial. For example, we provide the following equation system:

$$
-i \frac{d}{d t} C_{J M}(t)=\frac{\gamma}{2}\left[\exp \left\{4 \pi i t \frac{\Delta B}{B_{\mathrm{f}}} J\right\} C_{J-1, M}(t)+\exp \left\{-4 \pi i t \frac{\Delta B}{B_{\mathrm{f}}}(J+1)\right\} C_{J+1, M}(t)\right] .
$$

This equation was obtained by substituting $D_{\mathrm{v}}=0$ and $\sqrt{\omega_{j}}=\frac{\gamma}{2}$ into Eq. (2.5). For the case of $\Delta B / B_{\mathrm{f}}=0.00$, the rotational distribution was rapidly dispersed into large $J$ states [Fig. 1(a)]. Moreover, no localization was observed. For the case of $\Delta B / B_{\mathrm{f}}=0.03$, the population hardly transfers from the initial state [Fig. 1(b)]. This is the strong localization caused by interval mismatching. The approximate same strong localization can be observed from any initial state [Fig. 1(c)].

\subsection{Localization 2: Localization by Centrifugal Distortion}

Because the real molecule is not a rigid rotor, the centrifugal distortion significantly influences the evolution of rotational distribution. Despite the case of $\Delta B / B_{\mathrm{f}}=0$, a weak localization will be observed for $D_{\mathrm{v}} / B_{\mathrm{f}} \neq 0$. In real systems, the order of magnitude of $\frac{D_{\mathrm{v}}}{B_{\mathrm{f}}} \approx \frac{D_{\mathrm{v}}}{B_{\mathrm{M}}}$ is generally about $10^{-5}-10^{-7}$; therefore, the observed localization 


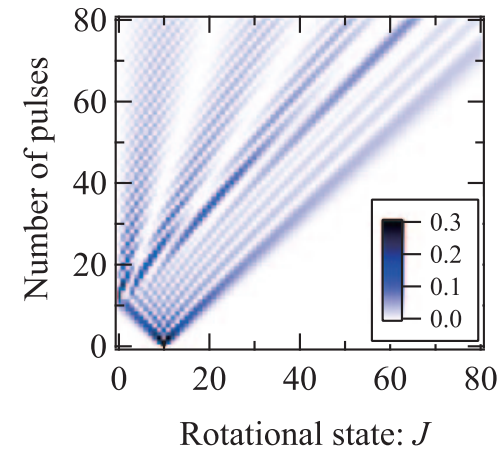

(a)

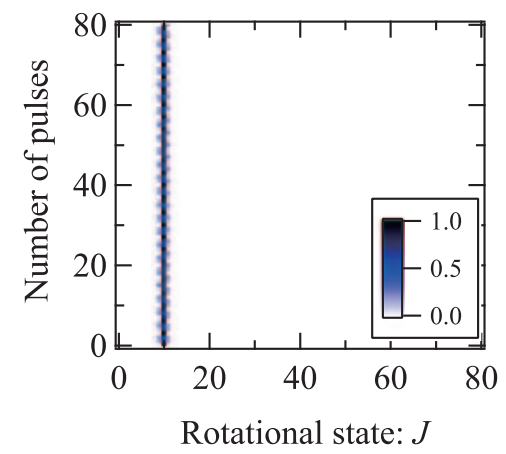

(b)

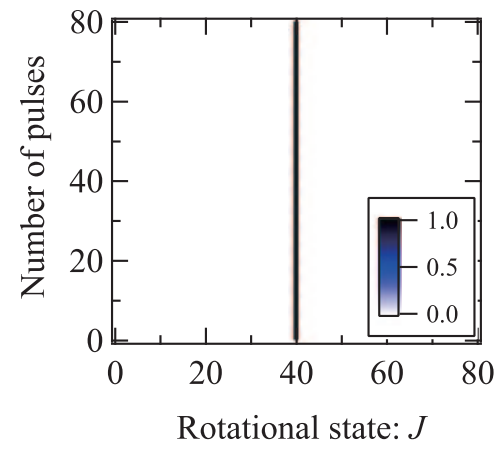

(c)

Fig. 1. Examples of time evolutions of Eq. (3.1) simulated with $\gamma=2.0$ and $\Delta B / B_{\mathrm{f}}=0.00$ from $J=10$ (a), $\gamma=2.0$ and $\Delta B / B_{\mathrm{f}}=0.03$ from $J=10(\mathrm{~b})$, and $\gamma=2.0$ and $\Delta B / B_{\mathrm{f}}=0.03$ from $J=40$ (c).

becomes weaker than that by interval mismatching, even though $D_{\mathrm{v}}$ is multiplied by the terms of $J^{3}$. This weak localization is called Bloch oscillation in a similar context [1]. The range of localization exhibits nontrivial dependence on the amplitude of pulses, the same as localization by $\Delta B$. For example, consider the equation:

$$
-i \frac{d}{d t} C_{J M}(t)=\frac{\gamma}{2}\left[\exp \left\{-8 \pi i t \frac{D_{\mathrm{v}}}{B_{\mathrm{f}}} J^{3}\right\} C_{J-1, M}(t)+\exp \left\{8 \pi i t \frac{D_{\mathrm{v}}}{B_{\mathrm{f}}}(J+1)^{3}\right\} C_{J+1, M}(t)\right] .
$$

This equation was obtained by substituting $\Delta B=0$ and $\sqrt{\omega_{j}}=\frac{\gamma}{2}$ into Eq. (2.5). Figure 2 shows an example of time evolution with weak localization by centrifugal distortion. For the case of weak localization, the turning point always appears nearly in the same position, regardless of the initial state [Figs. 2(a) and 2(b)]. For an initial state above the turning point, the time evolution is similar to the localization by interval mismatching [Fig. 2(c)]. The dependence of the range of localization on the pulse amplitude is shown in Fig. 3. This localization can be canceled by adjusting the phase of the optical pulses; however, it is experimentally difficult to obtain using current technologies.

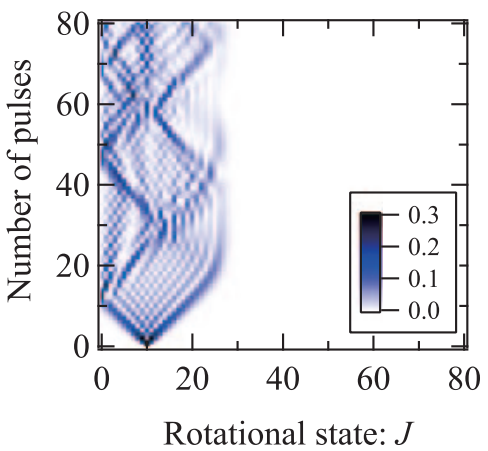

(a)

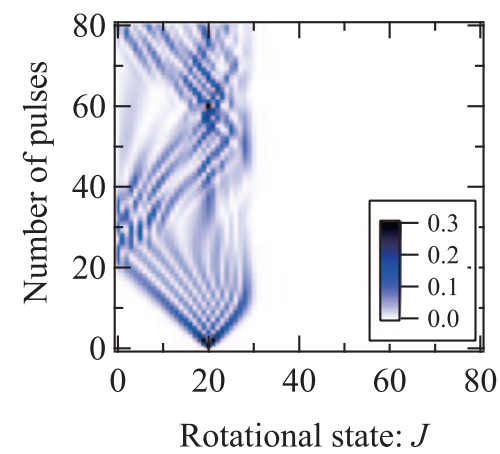

(b)

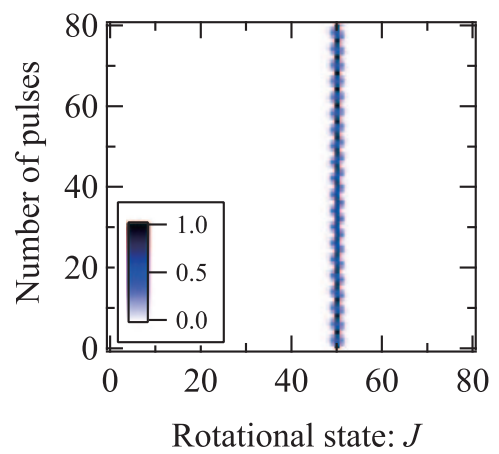

(c)

Fig. 2. Examples of time evolutions of Eq. (3.2) simulated with $\gamma=2.0$ and $D_{\mathrm{v}} / B_{\mathrm{f}}=1.0 \times 10^{-6}$ from $J=10$ (a), $J=20$ (b), and $J=50(\mathrm{c})$.

\subsection{Localization 3: Localization by Lack of Spectral Amplitude}

In real systems, the transition probability to a neighboring state becomes zero in a highly excited state because the linewidth of a real optical pulse is finite. If the transition probability suddenly falls from a constant value to zero, the reflection of the population would be observed at that state. Actually, such a reflection can be observed at $J=0$. However, in the case that the transition probability asymptotically approaches zero, predicting the point of reflection is difficult. To our knowledge, the detail of this type of localization is not explicitly discussed elsewhere. In the following, we give an example of Gaussian spectral amplitude that is peaked at $J=0$ :

$$
-i \frac{d}{d t} C_{J M}(t)=A_{g} \exp \left(-\frac{(J-1)^{2}}{B_{g}^{2}}\right) C_{J-1, M}(t)+A_{g} \exp \left(-\frac{J^{2}}{B_{g}^{2}}\right) C_{J+1, M}(t) .
$$

This equation was obtained by substituting $\Delta B=0, D_{\mathrm{v}}=0$, and $\sqrt{\omega_{j+1}}=A_{g} \exp \left(-j^{2} / B_{g}^{2}\right)$ into Eq. (2.5). The time evolutions of Eq. (3.3) for a same $B_{g}$ and different $A_{g}$ are shown in Fig. 4. It is worth to mention that the reflection 


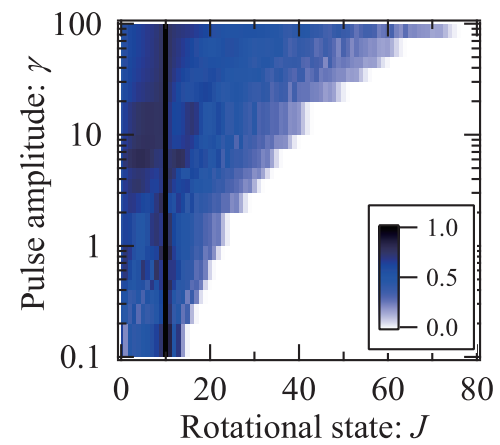

Fig. 3. Time-averaged relative population distribution using Eq. (3.2) with various $\gamma$ and $D_{\mathrm{v}} / B_{\mathrm{f}}=1.0 \times 10^{-6}$ from $J=10$.

behavior of these three cases is completely the same, except for the speed of evolution. The range of localization on this case is determined by the relative shape of spectral amplitude, and it does not depend on its absolute intensity.

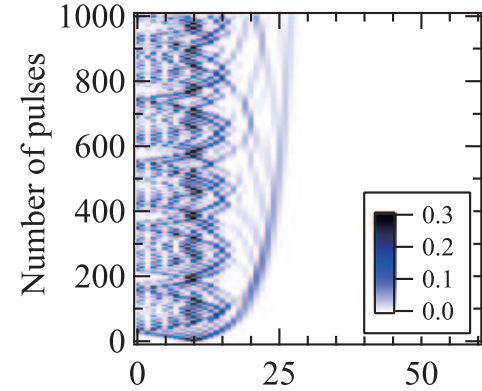

Rotational state: $J$

(a)

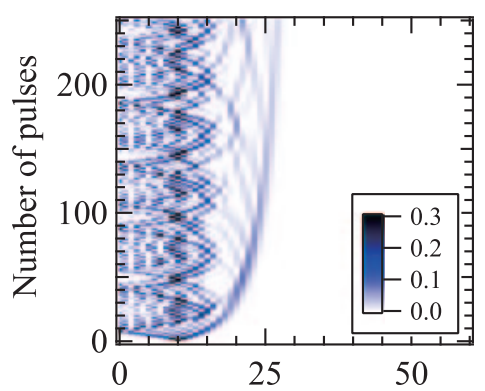

Rotational state: $J$

(b)

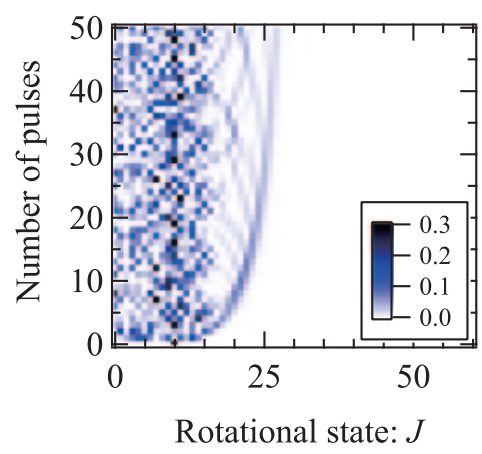

(c)

Fig. 4. Examples of time evolutions of Eq. (3.3) simulated with $B_{g} \approx 12.01$ with $A_{g}=0.25$ (a), $A_{g}=1.0$ (b), and $A_{g}=5.0$ (c), from $J=10$. The value of $B_{g}$ corresponds to $H W H M=10.0$.

\subsection{Localization 4: Localization by Augmentation of Spectral Amplitude}

In localization 3, we assumed that the transition probabilities, which come from the spectral amplitude of pulses, change smoothly on each state. When the spectral amplitude sharply changes, another type of localization will be observed at that point. This localization was predicted by Segawa et al. in their mathematical considerations. They named this phenomenon "localization by augmentation". For an extreme example, consider the equation:

$$
\begin{aligned}
-i \frac{d}{d t} C_{J M}(t) & =\sqrt{\omega_{J}} C_{J-1, M}(t)+\sqrt{\omega_{J+1}} C_{J+1, M}(t), \\
\omega_{j} & = \begin{cases}p & (j=1) \\
q & (j>1)\end{cases}
\end{aligned}
$$

This equation was obtained by substituting $\Delta B=0, D_{\mathrm{v}}=0$, and $\sqrt{\omega_{1}}=p, \sqrt{\omega_{j>1}}=q$ into Eq. (2.5). The results of population distribution after 100 pulses are shown in Fig. 5. For the case of $p<2 q$, no localization can be observed [Fig. 5(a)]. When $p>2 q$, part of the population was trapped around $J=0$ [Figs. 5(b) and 5(c)]. For this type of localization, the remaining part of the population was not localized, and escaped into highly excited states. This localization needs further studies. For example, the requirement for localization is not clear in the case where two or more augmentations are included in the system.

\section{Towards a Unified Approach for Predicting the Range of Localization}

In real systems, the localization will appear as a combination of the effects of the four localizations presented here. For a prediction of the range of localization, we need to consider all the four localizations simultaneously. Here, we briefly introduce our prospects towards a unified theoretical approach in which no numerical simulation is required. We have two approaches for the unified treatment of localization in this system. One is a semi-analytical approach with empirical parameters, and the other is a full analytical approach.

In the semi-analytical approach, we focus on whether the range of localization depends on the pulse amplitude or not. Localizations 1 and 2 depend on the amplitude, and localizations 3 and 4 are independent of it. We have already 


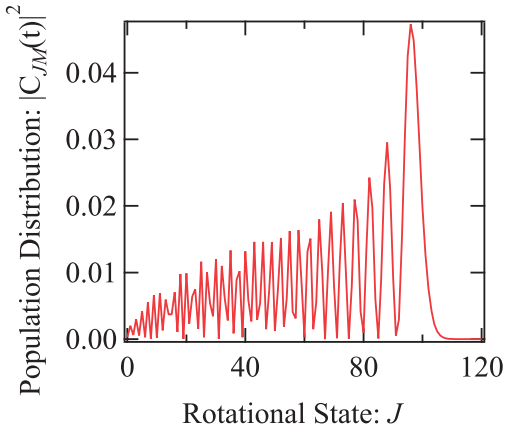

(a)

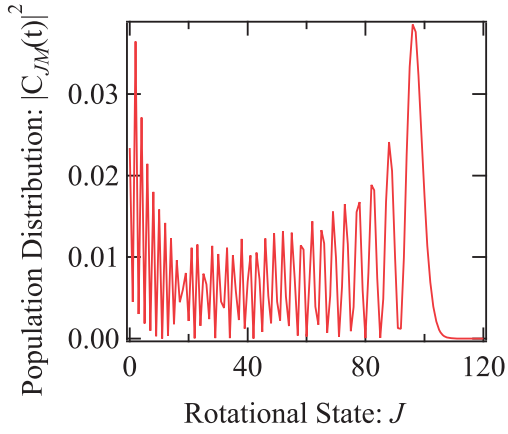

(b)

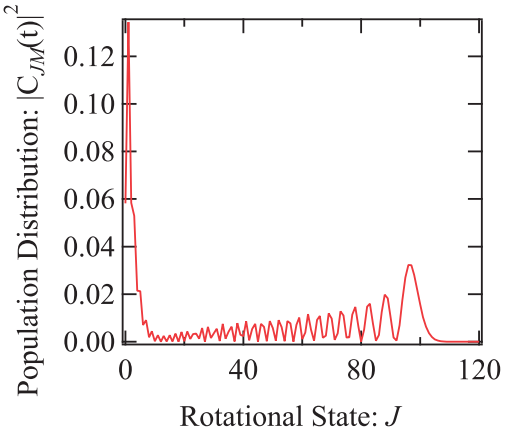

(c)

Fig. 5. Examples of population distribution after 100 pulses obtained by Eq. (3.4) from $J=0$ with $q=0.25$ and $p=0.45$ (a), $p=0.55(\mathrm{~b})$, and $p=0.65(\mathrm{c})$.

derived the unified parameter for the combination effect of localizations 1 and 2 [10]. The unified parameter comprises the peak amplitude and interval between the pulses as well as the level energies of the initial $(J=r)$ and final $(J=s)$ rotational states of the molecule. For the model equation system Eq. (2.5) with $\sqrt{\omega_{j}}=\frac{\gamma}{2}$, the unified parameter is given as

$$
u_{\mathrm{L}}=\frac{\gamma \cdot B_{\mathrm{f}}}{\left|\beta_{s}-\beta_{r}\right|}
$$

where $\beta_{J}$ is given by

$$
\beta_{J}=E_{J}-2 \pi B_{\mathrm{f}} J(J+1) .
$$

The population distribution is localized in the region of $u_{\mathrm{L}}>0.5$. Even though the relation between the unified parameter and the range of localization was obtained empirically, it aptly describes the dependence of the range of localization on the amplitude of pulses. Because localizations 3 and 4 do not depend on the amplitude of pulses, their effects should be expressed as correction factors for the unified parameter. Numerical evaluations for limited cases will determine the correlation factors, and they will be extended for general cases.

In the full analytical approach, we are attempting to formulate the asymptotic behavior of time evolution by using a method of orthogonal polynomials. The limit distribution of several simple cases for localizations 3 and 4 has been already formulated in our mathematical analysis. We are improving the mathematical foundation and making examples. This approach will be extended to localizations 1 and 2, and all the localization in rotational excitation in diatomic molecules will soon be predicted without numerical simulations.

\section{Acknowledgments}

We acknowledge the helpful discussions with Nobuaki Obata and Hayato Saigo. This work was supported by JSPS KAKENHI Grant No. JP26420875. ES's work is partially supported by the Japan-Korea Basic Scientific Cooperation Program "Non-commutative Stochastic Analysis: New Prospects of Quantum White Noise and Quantum Walk" (2015-2016).

\section{REFERENCES}

[1] Floß, J., and Averbukh, I. Sh., "Anderson wall and Bloch oscillations in molecular rotation," Phys. Rev. Lett., 113: 043002 (2014).

[2] Izrailev, F. M., and Shepelyanskii, D. L., "Quantum resonance for a rotator in a non-linear periodic field," Theor. Mathemat. Phys., 43: 553-561 (1980).

[3] Fleischer, S., Averbukh, I. Sh., and Prior, Y., "Isotope-selective laser molecular alignment," Phys. Rev. A, 74: 041403 (2006).

[4] Zhdanovich, S., Bloomquist, C., Floß, J., Averbukh, I. Sh., Hepburn, J. W., and Milner, V., "Quantum resonances in selective rotational excitation of molecules with a sequence of ultrashort laser pulses," Phys. Rev. Lett., 109: 043003 (2012).

[5] Akagi, H., Kasajima, T., Kumada, T., Itakura, R., Yokoyama, A., Hasegawa, H., and Ohshima, Y., "Isotope-selective ionization utilizing field-free alignment of isotopologues with a train of femtosecond laser pulses," Phys. Rev. A, 91: 063416 (2015).

[6] Yokoyama, K., Matsuoka, L., Kasajima, T., Tsubouchi, M., and Yokoyama, A., "Quantum control of molecular vibration and rotation toward the isotope separation," Advances in Intense Laser Science and Photonics, edited Lee J. et al. (Publishing House for Science and Technology, Hanoi, 2010: 113-119 (2010).

[7] Matsuoka, L., Ichihara, A., Hashimoto, M., and Yokoyama, K., "Theoretical study for laser isotope separation of heavyelement molecules in a thermal distribution," Proceedings of the International Conference Toward and Over the Fukushima Daiichi Accident (GLOBAL 2011), Makuhari, Japan, Paper No. 392063 (CD-ROM) (2011). 
[8] Ichihara, A., Matsuoka, L., Segawa, E., and Yokoyama, K., "Isotope-selective dissociation of diatomic molecules by terahertz optical pulses," Phys. Rev. A, 91: 043404 (2015).

[9] Matsuoka, L., Kasajima, T., Hashimoto, M., and Yokoyama, K., "Numerical study on quantum walks implemented on cascade rotational transitions in a diatomic molecule," J. Korean Phys. Soc., 59: 2897-2900 (2011).

[10] Matsuoka, L., "Unified parameter for localization in isotope-selective rotational excitation of diatomic molecules using a train of optical pulses," Phys. Rev. A, 91: 043420 (2015). 